\title{
Circulating endothelial precursors: mystery, reality, and promise
}

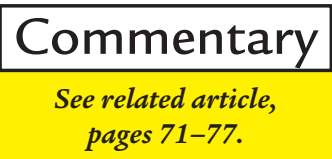

\author{
Shahin Rafii \\ Weill Medical College of Cornell University, New York, New York 10021, USA. \\ Phone: (212) 746-2070; Fax: (212) 746-8866; E-mail: srafii@mail.med.cornell.edu.
}

Postnatal physiological and pathophysiological processes such as wound healing and tumor growth require endothelial migration, proliferation, and stabilization, a process referred to as angiogenesis. Initial phases of angiogenesis involve the recruitment of endothelial cells to the site of injury or to the tumor vascular bed. Two possible sources of endothelialization are (a) migration and co-option $(1,2)$ of preexisting vascular wall endothelial cells and (b) recruitment of endothelial precursor cells from the circulation (Figure 1). There is ample evidence for the first scenario. However, the existence of angioblast-like circulating endothelial precursor cell (CEP) in adult humans has only recently been suggested, and their role in supporting postnatal angiogenesis is under intensive investigation.

CEP may reflect the phenotype of embryonic angioblasts, which are migratory endothelial cells with the capacity to circulate, proliferate, and differentiate into mature endothelial cells, but which have neither acquired characteristic markers of mature endothelium nor formed lumina $(3,4)$. Although there is abundant evidence of the existence of angioblasts during embryonic development, the isolation and characterization of angioblast-like CEPs from adult circulation has been hampered by the absence of specific endothelial markers and functional assays to distinguish angioblast-like CEPs from sloughed mature endothelial and hematopoietic cells.

Both putative CEPs with angioblastic potential and vessel wall-derived mature endothelial cells may express similar endothelial-specific markers, including vascular endothelial growth factor receptor-2 (VEGFR2, KDR, Flk1) (5), Tie-2, Tie-1 $(6,7)$, VE-cadherin (8), CD34, and E-selectin (Table 1). Therefore, it may be impractical to use these markers to distinguish between the 2 populations. Discrimination between CEPs and mature endothelial cells is further complicated by the fact that subsets of hematopoietic cells express markers similar to those of endothelial cells such as CD34, PECAM (CD31), Tie-1, Tie-2, von Willebrand factor (vWF), and VEGFR2 (9). Furthermore, certain hematopoietic cells are also able to incorporate acetylated LDL (AcLDL) and to bind lectins such as BS-1 and ulex europaeus, which are usually considered endothelial specific.

Several studies have attempted to identify CEPs by performing bone marrow (BM) transplantation studies in which recipient and donor cells could be distinguished by endothelialspecific markers. One study (10) has suggested that BM-derived VEGFR2+ endothelial progenitor cells could be detected in the peripheral circulation and contribute to vasculogenesis. However, although $27 \%$ of freshly iso-

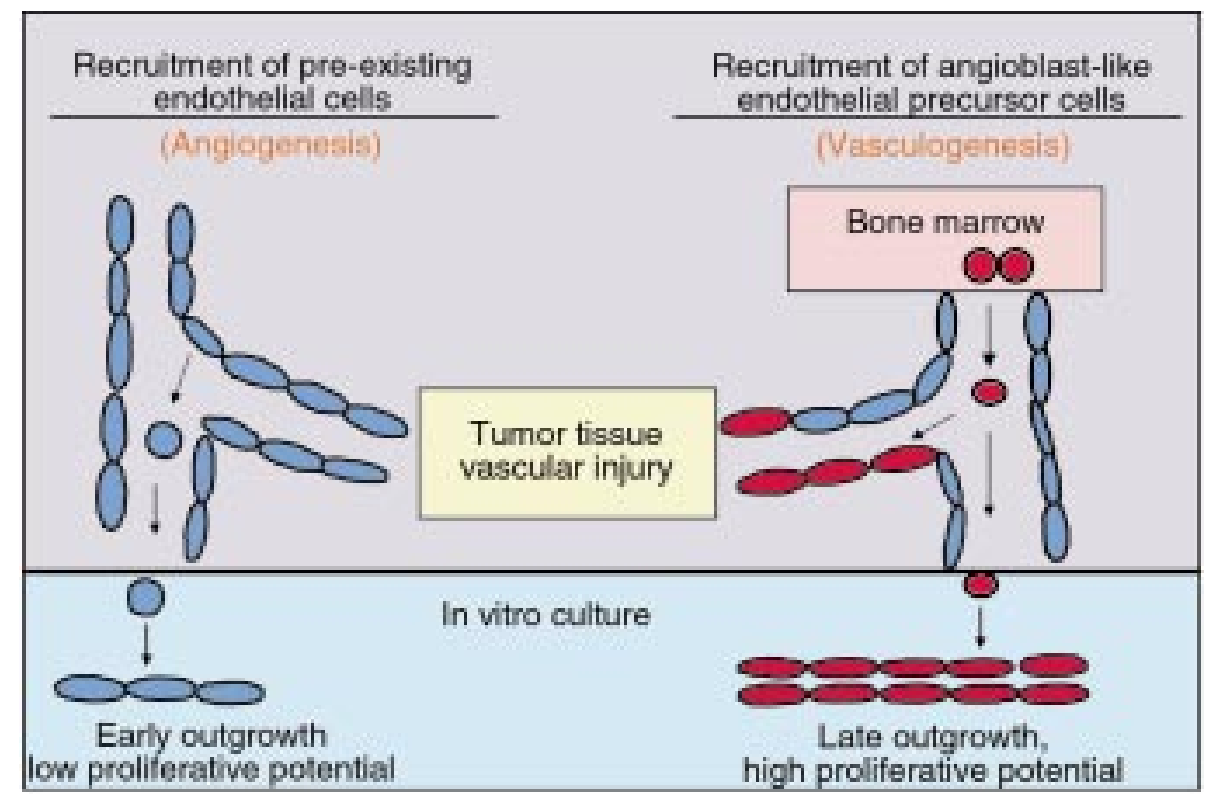

Figure 1

Models of postnatal angiogenesis and vasculogenesis. Recruitment of endothelial cells from preexisting vessel wall or CEPs plays a critical role in the regulation of postnatal angiogenesis and vasculogenesis. Mobilized BM-derived CEPs with high proliferative capacity may have the potential to home and incorporate into the injured tissue or tumor vascular bed. 
Table 1

Phenotypic analysis of putative endothelial precursor cells

\begin{tabular}{|c|c|c|c|}
\hline Surface markers & $\begin{array}{c}\text { Putative Endothelial } \\
\text { precursor cell }\end{array}$ & $\begin{array}{c}\text { Vessel wall } \\
\text { endothelium }\end{array}$ & Hematopoietic cells \\
\hline VEGFR-2 (KDR, Flk-1) & + & + & Subset of stem cells \\
\hline VEGFR-3 (Flt-4) & ? & + & $?$ \\
\hline VEGFR-1 (Flt-1) & $?$ & + & + \\
\hline Tie-2 & $?$ & + & + \\
\hline VE-cadherin & + & + & - \\
\hline CD34 & + & + & Stem and progenitor cells \\
\hline Ac.LDL uptake & + & + & + \\
\hline PECAM (CD31) & + & + & + \\
\hline AC133 & + & - & Subset of stem cells \\
\hline
\end{tabular}

lated $\mathrm{CD}_{34}{ }^{+}$cells were VEGFR2 ${ }^{+}$as determined by flow cytometry, the authors of this study were not able to detect VEGFR2 mRNA message in the first 7 days of culture using RT-PCR, perhaps because the isolated cells were not, in fact, of endothelial origin. This concern arises because of the use of an antibody that was specific to the intracellular, rather than the extracellular, domain of VEGFR2. In subsequent studies, populations of cells enriched for endothelial progenitor cells were derived from murine peripheral blood cells or BM and labeled with DiI to track the mobilization and recruitment of these cells into ischemic limbs $(11,12)$. However, given that subsets of hematopoietic cells have the capacity to express CD31, Tie-2, and incorporate AcLDL, it is not clear whether the mobilized cells and the cells incorporated into the vessel wall were of primarily hematopoietic or endothelial origin.

We have shown that allogeneic sexmismatched BM transplantation results in the transfer of endothelial cells to recipient dogs (13). Replacement of the aorta of the recipient dogs months after transplantation with impervious Dacron grafts resulted in graft endothelialization arising exclusively from the transplanted BM. In humans, evidence for CEPs originates from patients implanted with a left ventricular assist device (LVAD). We have demonstrated colonization of the flow surface of the titanium housing of LVADs with $\mathrm{CD}_{3} 4^{+}$endothelial-like cells 6 months after the devices were removed (14). These studies suggest that transplanted CEPs residing in the BM have the capacity to be mobilized to the peripheral circulation and incorporate in the sites of active angio- genesis. However, because transplantation of whole BM results in transfer of both mature BM endothelium and putative angioblast-like CEPs, none of these studies have conclusively demonstrated the existence of a phenotypically and functionally distinct population of CEPs.

The article by Lin et al. in this issue capitalizes on the in vitro expansion potential and growth kinetics of endothelial cells outgrown from peripheral blood mononuclear cells to discriminate between BM versus vessel wall-derived endothelial cells (15). Using fluorescent in situ hybridization analysis to detect the $\mathrm{Y}$ chromosome in blood samples from BM-transplant recipients who received gender-mismatched stem cells, Lin et al. can distinguish between vessel wall- and BMderived endothelial cells. They show that in vitro-derived endothelial cells from early phases ( 9 days after culture) undergo only 6 -fold expansion and are derived predominantly from the recipient vessel wall, whereas endothelial cells derived from late-outgrowth cells (27 days after culture) undergo 98-fold expansion and mostly originate from transplanted donor BM cells.

Based on these results, it is logical to speculate that, as with hematopoietic progenitor cells, late-outgrowth endothelial cells enjoy a relatively large capacity to proliferate and therefore can act as precursor cells in this lineage. Therefore, these cells may be considered as angioblast-like endothelial cells. The difference in growth might also be ascribed to differences in cell cycle status or, if donor and receptor animals are mismatched for age, to differences in telomere length between donor- and recipient-derived cells. However, the remarkable reproducibility of the results reported by Lin et al. strongly suggest that late-outgrowth endothelial cells that are derived from transplanted BM cells have different proliferative potential from those of the native vessel-derived endothelial cells.

One difficulty with the methodology presented by Lin et al. is the paucity of CEPs in the starting samples and the long culture period necessary to identify the late-outgrowth cells. In this regard, identification of a novel surface marker to distinguish between CEPs with progenitor potential and vessel wall-derived endothelial cells could significantly enhance our ability to isolate and study these cells in vitro. One such candidate marker is AC133, a novel hematopoietic stem cell marker (16), which is also expressed on subsets of CEPs, but not on mature endothelial cells (17). We have shown that a small subset of $\mathrm{CD} 34^{+}$cells derived from different hematopoietic sources express both AC133 and VEGFR2. Maturation and in vitro differentiation of these cells results in the loss of AC133 expression, suggesting that CEP with angioblast potential may be marked selectively with AC133.

During the last 2 decades, the emergence of specific surface markers and molecular probes has facilitated the identification and purification of functional hematopoietic stem and progenitor cells. Similarly, the identification of unique surface markers and functional assays, such as those proposed by Lin et al., will undoubtedly increase our ability to purify and dissect the biological potential of CEPs. Development of ex vivo expansion techniques for angioblast-like CEPs will be useful to promote vascular healing, provide suitable coating for vascular grafts, or deliver lethal toxins to tumor vascular beds. Moreover, identification of the chemokines or other biological cues that may induce mobilization of CEPs from BM to the peripheral circulation may provide a novel mechanism to recruit these cells to sites of vascular trauma and to accelerate vascular healing. Genetically modified autologous or allogeneic CEPs may provide ideal vehicles to deliver genes selectively to the vessel wall. Generation of neutralizing antibodies to functional receptors on CEPs will increase our understanding of whether recruitment and incorporation of CEPs to the tumor vascular 
bed are essential for tumor progression and metastasis. Finally, more sophisticated purification and transplantation techniques will allow us to determine the contribution of these unique endothelial cells to postnatal angiogenesis and vasculogenesis.

1. Holash, J., et al. 1999. Vessel cooption, regression, and growth in tumors mediated by angiopoietins and VEGF. Science. 284:1994-1998.

2. Hanahan, D., and Folkman, J. 1996. Patterns and emerging mechanisms of the angiogenic switch during tumorigenesis. Cell. 86:353-364.

3. Coffin, J.D., Harrison, J., Schwartz, S., and Heimark, R. 1991. Angioblast differentiation and morphogenesis of the vascular endothelium in the mouse embryo. Dev. Biol. 148:51-62.

4. Caprioli, A., Jaffredo, T., Gautier, R., Dubourg, C., and Dieterlen-Lievre, F. 1998. Blood-borne seeding by hematopoietic and endothelial precursors from the allantois. Proc. Natl. Acad. Sci. USA. 95:1641-1646.
5. Eichmann, A., et al. 1997. Ligand-dependent development of the endothelial and hemopoietic lineages from embryonic mesodermal cells expressing vascular endothelial growth factor receptor 2. Proc. Natl. Acad. Sci. USA 94:5141-5146

6. Sato, T.N., Qin, Y., Kozak, C.A., and Audus, K.L. 1993. Tie-1 and Tie-2 define another class of putative receptor tyrosine kinase genes expressed in early embryonic vascular system [erratum 1993, 90:12056]. Proc. Natl. Acad. Sci. USA. 90:9355-9358.

7. Suri, C., et al. 1996. Requisite role of angiopoietin-1, a ligand for the TIE2 receptor, during embryonic angiogenesis. Cell. 87:1171-1180.

8. Vittet, D., et al. 1996. Embryonic stem cells differentiate in vitro to endothelial cells through successive maturation steps. Blood. 88:3424-3431.

9. Ziegler, B.L., et al. 1999. KDR receptor: a key marker defining hematopoietic stem cells. Science. 285:1553-1558.

10. Asahara, T., et al. 1997. Isolation of putative progenitor endothelial cells for angiogenesis. Science. 275:964-967.

11. Takahashi, T., et al. 1999. Ischemia- and cytokine- induced mobilization of bone marrow-derived endothelial progenitor cells for neovascularization. Nat. Med. 5:434-438.

12. Asahara, T., et al. 1999. VEGF contributes to postnatal neovascularization by mobilizing bone marrow-derived endothelial progenitor cells. EMBO J. 18:3964-3972.

13. Shi, Q., et al. 1998. Evidence for circulating bone marrow-derived endothelial cells. Blood. 92:362-367.

14. Rafii, S., et al. 1995. Characterization of hematopoietic cells arising on the textured surface of left ventricular assist devices. Ann. Thorac Surg. 60:1627-1632.

15. Lin, Y., Weisdorf, D., Solovey, A., and Hebbel, R 1999. Origins of circulating endothelial cells and endothelial outgrowth from blood. J. Clin. Invest. 105:71-77

16. Yin, A.H., et al. 1997. AC133, a novel marker for human hematopoietic stem and progenitor cells. Blood. 90:5002-5012.

17. Peichev, M., et al. 2000. Expression of VEGFR-2 and $\mathrm{AC} 133$ by circulating human $\mathrm{CD} 34^{+}$cells identifies a population of functional endothelial precursors. Blood. In press. 